\title{
Validity of the Semiclassical Kinetic Energy Density Functional for Deformed Nuclear Shapes
}

\author{
C. Guet ${ }^{\star}$ and M. Brack \\ Institut für Theoretische Physik der Universität, Regensburg, \\ Federal Republic of Germany
}

Received March 24, 1980; revised version June 19, 1980

\begin{abstract}
We investigate the semiclassical kinetic energy density functional $\tau[\rho]$ for deformed nuclear shapes. Using Strutinsky-averaged quantities as reference, we demonstrate that the functional $\tau[\rho]$ is able to reproduce the averaged kinetic energies very accurately, even at very large deformations.
\end{abstract}

\section{Introduction}

The use of semiclassical methods in calculations of nuclear bulk properties like binding and deformation energies, density distributions and radii [1] or high lying collective excitations $[2,3]$ have recently gained a renewed interest. Model Hartree-Fock calculations using Strutinsky-smoothed densities [4] have, in fact, given us a strong motivation for the treatment of the static selfconsistent nuclear problem in a semiclassical approximation; shell effects may be added perturbatively when the selfconsistency is reached for the average nuclear densities and potentials.

Effective nucleon-nucleon interactions of the Skyrme type [5] are especially well suited for semiclassical calculations: for these, the nuclear binding energy in the Hartree-Fock approximation can be expressed directly in terms of the local spatial and kinetic energy densities $\rho(r)$ and $\tau(r)$, respectively. The latter is usually defined as

$\tau(r)=\sum_{i=1}^{N(Z)}\left|\boldsymbol{V} \psi_{i}(r)\right|^{2}$

in terms of the (neutron or proton) single particle wave functions $\psi_{i}(r)$. With the help of an explicit functional $\tau[\rho]$ expressing the kinetic energy density in terms of the spatial density, the total nuclear binding energy is then given entirely by the densities $\rho_{n}(r)$ and $\rho_{p}(r)$ and their gradients. A variational calculation can thus be performed to determine the

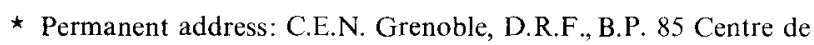
Tri, F-38041 Grenoble Cedex, France best densities $\rho_{n}(r)$ and $\rho_{p}(r)$. Although Hohenberg and Kohn [6] proved the basic existence of a universal functional $\tau[\rho]$, there is little hope that a simple form of $\tau[\rho]$ will be sufficient in this procedure to lead to the exact (Hartree-Fock) energy. However, when dealing with average densities and binding energies only, thus ignoring shell effects, good results were obtained for spherical nuclei [1] with the semiclassical functional $\tau[\rho]$ as obtained within the framework of the extended Thomas-Fermi (ETF) model $[7,8]$. (We do not discuss here older versions of the so-called energy density formalism [9] with or without Weizsäcker correction, in which errors in the kinetic energy were renormalized together with uncertainties in the potential nuclear energy with the help of adjustable phenomenological parameters.)

It is the aim of the present paper to demonstrate that the ETF density functional $\tau[\rho]$ can be applied to deformed nuclear shapes as well as to spherical ones. Before we do so, let us briefly recall some essential features of the ETF model and the derivation of the corresponding semiclassical functional $\tau[\rho]$.

\section{The Semiclassical Functional $\tau[\rho]$}

In the ETF model for independent Fermions moving in a given (e.g. Hartree-Fock) potential $V(r)$, semiclassical corrections to the familiar TF expressions for the densities $\rho_{\mathrm{TF}}(r)$ and $\tau_{\mathrm{TF}}(r)$ are obtained by 
means of an expansion in powers of $\hbar$ (see e.g., $[7,8]$ ):

$\rho_{\mathrm{ETF}}=\rho_{\mathrm{TF}}+\rho_{2}+\rho_{4}+\ldots ; \quad \tau_{\mathrm{ETF}}=\tau_{\mathrm{TF}}+\tau_{2}+\tau_{4}+\ldots$

here the indices 2 and 4 denote the order of $\hbar$ in the corresponding terms. The densities (2) are only defined in the classically allowed region; they both diverge at the turning point due to the semiclassical corrections. Nevertheless, integrated quantities such as particle number and potential or kinetic energy are well defined. Furthermore, the total ETF energies obtained for various types of nuclear potentials converge within $\sim 1 \mathrm{MeV}$ if terms up to order $\hbar^{4}$ are retained; their values also agree with the Strutinskyaveraged energies within $\lesssim 1-2 \mathrm{MeV}$ in all cases $[8,11]$ (see also below).

An explicit expression for the functional $\tau[\rho]$ is now easily obtained by algebraic elimination of the potential $V(r)$ and its derivatives from the explicit expressions for $\tau_{E T F}$ and $\rho_{\text {ETF }}(2)$. The result, which holds for any local potential $V(r)$, is $[7,8]$

$\tau_{\mathrm{ETF}}[\rho]=\tau_{\mathrm{TF}}[\rho]+\tau_{2}[\rho]+\tau_{4}[\rho]$,

where the indices again show the order of $\hbar$ up to which $\tau_{\text {ETF }}$ and $\rho_{\text {ETF }}$ have been calculated. The individual terms read

$$
\begin{aligned}
& \tau_{\mathrm{TF}}[\rho]=\frac{3}{5}\left(3 \pi^{2}\right)^{2 / 3} \rho^{5 / 3}, \\
& \tau_{2}[\rho]=\frac{1}{36} \frac{(\nabla \rho)^{2}}{\rho}+\frac{1}{3} \Delta \rho, \\
& \tau_{4}[\rho]=\frac{1}{4320}\left(3 \pi^{2}\right)^{-2 / 3} \rho^{1 / 3}\left[24 \frac{\Delta^{2} \rho}{\rho}-60 \frac{\nabla \rho \cdot \nabla(\Delta \rho)}{\rho^{2}}\right. \\
& -28\left(\frac{\Delta \rho}{\rho}\right)^{2}-14 \frac{\Delta(\nabla \rho)^{2}}{\rho^{2}}+\frac{280}{3} \frac{(\nabla \rho)^{2} \Delta \rho}{\rho^{3}} \\
& \left.+\frac{184}{3} \frac{\nabla \rho \cdot \nabla(\nabla \rho)^{2}}{\rho^{3}}-96\left(\frac{\nabla \rho}{\rho}\right)^{4}\right] .
\end{aligned}
$$

The term $\tau_{\mathrm{TF}}[\rho]$ gives, of course, the well known TF relation between $\tau$ and $\rho$ which is exact for an infinitely extended Fermion system. The first term in $\tau_{2}[\rho]$ is the so-called Weizsäcker term, however 9 times smaller than the original inhomogeneity correction by Weizsäcker [12]. In calculating the total kinetic energy from Eqs. $(3,4)$, i.e., by integrating $\tau[\rho(r)]$ over the whole space, the Laplacian in $\tau_{2}[\rho]$ does not contribute; similarly after partial integration of $\tau_{4}[\rho]$, only three terms remain, containing no higher than second derivatives of $\rho(r)$ :

$$
\begin{aligned}
& T_{\mathrm{ETF}}[\rho]=\frac{\hbar^{2}}{2 m} \int \tau_{\mathrm{ETF}}[\rho(r)] d^{3} r \\
& =\frac{\hbar^{2}}{2 m} \int d^{3} r\left\{\frac{3}{5}\left(3 \pi^{2}\right)^{2 / 3} \rho^{5 / 3}+\frac{1}{36} \frac{(\nabla \rho)^{2}}{\rho}\right.
\end{aligned}
$$

$$
\begin{aligned}
& +\frac{1}{6480}\left(3 \pi^{2}\right)^{-2 / 3} \rho^{1 / 3}\left[8\left(\frac{\nabla \rho}{\rho}\right)^{4}-27\left(\frac{\nabla}{\rho}\right)^{2} \frac{\Delta \rho}{\rho}\right. \\
& \left.\left.+24\left(\frac{\Delta \rho}{\rho}\right)^{2}\right]\right\} .
\end{aligned}
$$

(Note that (3)-(5) hold separately for each kind of nucleons!)

Concerning the validity of this functional, the following remarks are relevant:

1) Equations (3)-(5) have been derived from a semiclassical model; therefore the functional can only be expected to reproduce average kinetic energies using average density distributions. There is no reason to expect (5) to give the exact kinetic energy when the exact quantum-mechanical density $\rho(r)$ is used.

2) The coefficients in (4) are uniquely given and independent of the potential $V(r)$ as long as it is local (see point 7 below); there exists no adjustable parameter.

3) In applications to realistic finite systems, the functional cannot be extended beyond the term $\tau_{4}[\rho]$. In fact, the straightforward continuation including terms proportional to $\hbar^{6}$ in $\tau_{\mathrm{ETF}}(r)$ and $\rho_{\mathrm{ETF}}(r)$ would lead to a term which goes asymptotically like $\rho^{-1 / 3}(r)$ and thus diverges when the density falls exponentially to zero. Thus, $(3,4)$ have to be considered as an asymptotic expansion of the true functional $\tau[\rho]$, which must be truncated after the term $\tau_{4}[\rho]$. This is typical for semiclassical expansions and thus consistent with the nature of the underlying ETF model.

4) A priori, the functional $\tau[\rho](3,4)$ is only valid inside the classically allowed region, since the densities $\tau_{\text {ETF }}$ and $\rho_{\text {ETF }}$ from which it was derived, are not defined at and beyond the classical turning point. Practically, one makes however the assumption that it may be extrapolated to the whole space, when sufficiently realistic smooth densities $\rho(r)$ are inserted. This assumption needs, of course, to be tested with realistic densities.

5) No assumption at all was made in deriving (3-5) concerning the shape of the potential or of the corresponding density. Thus, the functional a priori holds equally well for deformed as for spherical potentials. This, as well as point 2 above, is just a consequence of the uniqueness of the exact functional $\tau[\rho]$, as proven by Hohenberg and Kohn [6].

6) A microscopical way to obtain semiclassical dendities $\tilde{\tau}$ and $\tilde{\rho}$ is using the Strutinsky averaging method which was shown to be equivalent to a semiclassical expansion $[4,8,11]$. It is shown in [21] that the Strutinsky averaging of a system of nonin- 
teracting quasiparticles can be formulated selfconsistently as a variational problem. For this variational semiclassical system, the Hohenberg-Kohn theorem can be shown to hold using similar arguments as in the extension to a Fermigas at finite temperature [22]. Therefore, the use of density functionals such as $\tau[\rho]$ in connection with semiclassical densities $\tilde{\rho}$ is justified.

7) For nonlocal potentials, such as e.g., spin-orbit or effective mass terms, additional corrections to (3)-(5) must be included. They were derived in $[8,13]$ and will not be discussed further here.

8) The functional $\tau[\rho](3,4)$ is only valid for the relation between the static densities $\rho$ and $\tau$. In a time dependent treatment, additional terms arise in general which may be expressed in terms of a velocity field [2] and which must be explicitly taken into account in dynamical semiclassical calculations $[2,3]$. They play no role, however, in the calculation of static average deformation energies [14]. The latter may be obtained using a constraint on some multipole moment; this does not affect the validity of the functional, as long as the constraint is a function of coordinates only and thus can be added to the local potential (see also [23]).

Concerning point 8) above, there has been some confusion in the literature as to the exact conditions under which the semiclassical functional $\tau[\rho]$ may be applied to deformed nuclei. A clarification of this, based on the scaling argument of $[3,10]$, has now been put forward by Jennings [23]. Consider a deformed system for which the wavefunctions scale as

$\psi_{i}(x, y, z ; \lambda)=\psi_{i}\left(\lambda x, \lambda y, z / \lambda^{2}\right)$

Then the exact kinetic energy has the following dependence on the deformation parameter $\lambda$ (see also [17]):

$T(\lambda)=\frac{\hbar^{2}}{2 m} \int \tau(x, y, z ; \lambda) d^{3} r=\frac{1}{3} T_{0}\left(2 \lambda^{2}+\lambda^{-4}\right)$,

where $T_{0}=T(\lambda=1)$. It was correctly stated in $[3,10]$ that the semiclassical functional $\tau_{\mathrm{TF}}[\rho]+\tau_{2}[\rho]$ (even with readjusted coefficients) is not able to reproduce the kinetic energy $T(\lambda)(7)$. The reason for this breakdown is however, as discussed by Jennings [23], not the deformation in $r$-space, but that of the Fermi sphere in momentum space. Such a deformation in momentum space is typical e.g., for dynamical applications [2] in which the static functional is not supposed to apply (see point 8 above). For the axially deformed harmonic oscillator potential considered in Sect. III below, the deformation behavior $T(\lambda)(7)$ is exact also for the static quantum-mechanical kinetic energy in the case of a filled spherical shell. However - as stated above - it cannot be the purpose of a semiclassical functional to reproduce the exact quantum-mechanical kinetic energy. The point is that, even for a situation where $(6,7)$ are true, the semiclassical density $\tilde{\rho}$ will not deform as (6), and therefore the semiclassical kinetic energy $\tilde{T}(\lambda)$ will be different from (7). After all, it is well known that the deformation behaviour of a semiclassical, i.e., liquid-drop like system is locally completely different from that of a quantum mechanical system which includes shell effects. Thus, the above scaling argument cannot be used against the application of the semiclassical functional to statically deformed semiclassical systems,

The functional $\tau[\rho](3,4)$ including only $\tau_{\mathrm{TF}}$ and $\tau_{2}[\rho]$ has been used by various authors in variational calculations $[1-3]$. As pointed out by Berg and Wilets [15] and discussed later again [9], the inclusion of the Weizsäcker term is necessary in order to obtain solutions of the corresponding Euler-Lagrange equation for the density $\rho(r)$, which have an exponential fall-off for $r \rightarrow \infty$. With use of the TF-relation $\tau_{\mathrm{TF}}[\rho]$ only, the density has to be cut at a finite distance [15]. But even with inclusion of the Weizsäcker term, the fall-off of the variational density $\rho(r)$ is too fast; an increased Weizsäcker term by a factor $\sim 4-9$ leads to more realistic tails of $\rho(r)$. Therefore, several authors proposed to use phenomenological functionals in which the TF and Weizsäcker terms are multiplied by adjustable parameters $[10,15,16]$; the latter e.g., being determined by fits of the kinetic energies obtained through the functional $\tau[\rho]$ to Hartree-Fock results $[10,16]$.

We want to make here strong point that such a phenomenological adjustment of parameters is not necessary if the full functional $\tau[\rho]$ given by (3), (4) is used; in fact, as we will show, this allows to reproduce the average kinetic energies much more accurately than with the phenomenological functionals of $[10,16]$. Furthermore, the latter completely break down for deformed nuclear shapes, whereas this is not the case for (5).

The importance for the 4th order terms in $\tau[\rho](3-5)$ has already been pointed out in $[8]$ for a realistic Woods-Saxon potential at various deformations. It was shown there, that the total average kinetic energy (for neutrons and protons) of a heavy nucleus is reproduced within $\leqslant 2-4 \mathrm{MeV}$, i.e., within less than $1 \%$, whereas leaving out the $\tau_{4}[\rho]$ term (and keeping the coefficient $1 / 36$ of the Weizsäcker term) one would miss $\sim 20-30 \mathrm{MeV}$ or an amount of the order of $\sim 1 \%$. For completeness, we quote here some results of [8] in Fig. 1. It is seen, that not only the correct total kinetic energy $\tilde{T}$ (obtained by Strutinsky-smoothing of the exact $\tau(r))$ is reproduced within $\sim 1 \%$, but 


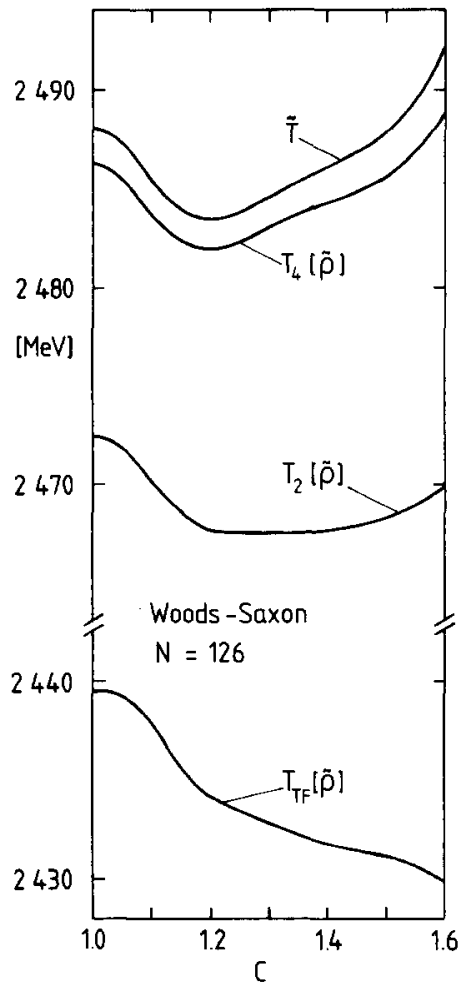

Fig. 1. Comparison of the semiclassical kinetic energy as delivered by means of the functional $\tau[\rho]$ for different orders in the expansion of $h$ (see text) to the Strutinsky smoothed kinetic energy $\tilde{T}$ for a system of 126 particles in a deformed Woods-Saxon potential. The deformation parameter $c$ measures the longer semi-axis in units of the radius $R_{0} . c=1$ corresponds to spherical shape and $c \approx 1.5$ to the saddle point on the liquid drop path for actinide nuclei. (Details see [19])

also its deformation behaviour is correct, in that the remaining error of $\sim 2-3 \mathrm{MeV}$ is almost constant with deformation.

In the present work, we want to continue and complete the investigations started in [8], using the Strutinsky-smoothed densities $\tilde{\tau}(r)$ and $\tilde{\rho}(r)$ of a deformed harmonic oscillator potential as the reference average densities for which the functional $\tau[\rho]$ can be tested. In particular, we want to emphasize here the extension to very large deformations.

\section{Test with Harmonic Oscillator Potential}

For our numerical investigations we shall consider the axially deformed harmonic oscillator potential with frequency ratio $q=\omega_{\perp} / \omega_{z}$ :

$$
\begin{aligned}
& V(x, y, z)=\frac{m}{2}\left[\omega_{\perp}^{2}\left(x^{2}+y^{2}\right)+\omega_{z}^{2} z^{2}\right] ; \\
& \omega_{\perp}=\omega_{0} q^{1 / 3} ; \quad \omega_{z}=\omega_{0} q^{-2 / 3} .
\end{aligned}
$$

For this potential, the Strutinsky-averaged part of the sum of $N$ occupied levels is known analytically [18] and is also identical with the ETF value of the energy (see $[8,11]$ ):

$$
\begin{aligned}
& \tilde{E}_{\mathrm{STRUT}}=E_{\mathrm{ETF}} \\
& =\frac{1}{4} \hbar \omega_{0}(3 N)^{4 / 3}\left[1+\frac{1}{2} r(q)(3 N)^{-2 / 3}+\mathcal{O}(3 N)^{-4 / 3}\right],
\end{aligned}
$$

where the deformation dependence is governed by the function

$r(q)=\frac{1}{3}\left(2 q^{2 / 3}+q^{-4 / 3}\right)$.

(The term of order $(3 N)^{-4 / 3}$ neglected in (9) contributes less than $0.005 \hbar \omega_{0}$ to the total energy even at a frequency ratio $q=4$.)

To test the semiclassical functional $\tau[\rho]$ (4), we have first constructed the Strutinsky-averaged densities

$\tilde{\rho}(r)=\sum_{i}\left|\psi_{i}(r)\right|^{2} \tilde{n}_{i}$,
$\tilde{\tau}(r)=\sum_{i}\left|\nabla \psi_{i}(r)\right|^{2} \tilde{n}_{i}$.

The average occupation numbers $\tilde{n}_{i}$ were calculated in the usual way (see e.g., $[18,19]$ ) using a smoothing width

$\gamma=(0.9-1.2) \hbar \omega_{0}$.

Hereby the plateau condition [18] was carefully fulfilled:

$\left.\frac{\partial \tilde{E}_{\text {STRUT }}}{\partial \gamma}\right|_{\gamma_{0}}=0$.

This is important here, because if (14) is not exactly fulfilled, not the entire information about the Strutinsky averaging leading to $\tilde{E}_{\text {STRUT }}(9)$ is contained in the occupation numbers $\tilde{n}_{i}$ and then the densities $\tilde{\rho}(r)$ and $\tilde{\tau}(r)(11,12)$ do not give the correct average quantities upon integration.

By construction, the integration of $\tilde{\tau}(r)(12)$ now leads to the average part of the kinetic energy of $N$ particles, which due to the virial theorem is equal to one half of the total energy (9):

$\frac{\hbar^{2}}{2 m} \int \tilde{\tau}(r) d^{3} r=\tilde{T}=\frac{1}{2} \tilde{E}_{\text {STRUT }}$.

On the other hand, the averaged densities $\tilde{\rho}(r)$ (11) may be used to test the semiclassical functional $\tau[\rho]$ (4). The main quantity of interest is, of course, the kinetic energy obtained through this functional and given in (5). We have thus calculated this energy $T[\tilde{\rho}]$, using the Strutinsky-averaged densities $\tilde{\rho}(r)$, and compared it to $\tilde{T}(15)$ for various nucleon num- 
Table 1. The quantities $T[\tilde{\rho}](5), V_{\tau}[\tilde{\rho}](18)$ (both in $\mathrm{MeV}$ ) and $\left\langle r^{2}\right\rangle_{\bar{\tau}}(17)$ (in $\mathrm{fm}^{2}$ ), as obtained either by means of the functional $\tau[\tilde{\rho}]$ (4), or using the Strutinsky smoothed $\tilde{\tau}(r)(12)$ for 112 nucleons in an axially deformed harmonic oscillator potential with frequency ratio $q\left(=\omega_{1} / \omega_{z}\right)$

\begin{tabular}{lllllllll}
\hline$q$ & $\tilde{T}$ & $T[\tilde{\rho}]$ & $\Delta T$ & $\tilde{V}_{\tau}$ & $V_{\mathrm{\tau}}[\tilde{\rho}]$ & $\Delta V_{\tau}$ & $\left\langle r^{2}\right\rangle_{i}$ & $\left\langle r^{2}\right\rangle_{\tau}[\tilde{\rho}]$ \\
\hline 0.4 & $2,238.70$ & $2,238.84$ & 0.14 & 368.19 & 368.51 & 0.32 & 32.654 & 32.923 \\
0.7 & $2,228.78$ & $2,229.02$ & 0.24 & 373.41 & 373.64 & 0.23 & 26.420 & 26.614 \\
1.0 & $2,227.38$ & $2,227.49$ & 0.11 & 374.13 & 374.34 & 0.21 & 25.217 & 25.386 \\
1.3 & $2,228.03$ & $2,227.84$ & -0.19 & 373.76 & 373.95 & 0.19 & 25.961 & 26.136 \\
1.6 & $2,229.45$ & $2,229.53$ & 0.08 & 372.89 & 373.05 & 0.16 & 27.789 & 27.945 \\
1.9 & $2,231.08$ & $2,231.12$ & 0.04 & 372.16 & 372.36 & 0.20 & 30.214 & 30.377 \\
2.2 & $2,233.00$ & $2,232.99$ & -0.01 & 371.33 & 371.36 & 0.03 & 33.119 & 33.313 \\
2.5 & $2,234.21$ & $2,234.31$ & 0.10 & 370.34 & 370.77 & 0.43 & 36.407 & 36.572 \\
2.8 & $2,236.76$ & $2,236.81$ & 0.05 & 368.80 & 369.36 & 0.56 & 40.231 & 40.432 \\
3.1 & $2,238.46$ & $2,238.84$ & 0.38 & 367.98 & 368.57 & 0.59 & 44.019 & 44.138 \\
\hline
\end{tabular}

Table 2. The same as in Table 1 for $q=1$ and various nucleon numbers $N$

\begin{tabular}{rrrrrrrrr}
\hline \multicolumn{1}{r}{} & \multicolumn{1}{c}{$T$} & \multicolumn{1}{c}{$T[\tilde{\rho}]$} & \multicolumn{1}{c}{$\Delta T$} & \multicolumn{1}{c}{$\tilde{V}_{\tau}$} & $V_{\tau}[\tilde{\rho}]$ & $\Delta V_{\tau}$ & $\left\langle r^{2}\right\rangle_{\tilde{\tau}}$ & $\left\langle r^{2}\right\rangle_{\tau}[\tilde{\rho}]$ \\
\hline 4 & 28.45 & 28.41 & -0.04 & 0.62 & 0.62 & 0.0 & 14.054 & 16.401 \\
16 & 170.91 & 170.68 & -0.23 & 9.64 & 9.67 & 0.03 & 16.284 & 16.934 \\
28 & 356.29 & 355.90 & -0.39 & 28.00 & 28.04 & 0.04 & 18.044 & 18.448 \\
40 & 570.13 & 569.70 & -0.43 & 54.82 & 54.90 & 0.08 & 19.477 & 19.775 \\
52 & 806.27 & 805.88 & -0.39 & 89.65 & 89.73 & 0.08 & 20.699 & 20.960 \\
64 & $1,061.12$ & $1,061.00$ & -0.12 & 132.12 & 132.19 & 0.07 & 21.780 & 21.949 \\
76 & $1,332.20$ & $1,332.72$ & 0.52 & 181.99 & 182.14 & 0.15 & 22.750 & 22.980 \\
88 & $1,617.82$ & $1,617.72$ & -0.10 & 239.09 & 239.25 & 0.16 & 23.632 & 23.841 \\
100 & $1,916.56$ & $1,916.72$ & 0.16 & 303.17 & 303.41 & 0.23 & 24.453 & 24.637 \\
112 & 2.227 .38 & $2,227.49$ & 0.11 & 374.13 & 374.34 & 0.21 & 25.217 & 25.386 \\
124 & $2,549.40$ & $2,549.76$ & 0.36 & 451.95 & 452.09 & 0.14 & 25.930 & 26.099 \\
136 & $2,881.21$ & $2,881.88$ & 0.67 & 536.40 & 536.67 & 0.27 & 26.595 & 26.728 \\
148 & $3,224.25$ & $3,224.26$ & 0.01 & 627.24 & 627.45 & 0.21 & 27.244 & 27.388 \\
160 & $3,575.88$ & $3,575.67$ & -0.21 & 724.57 & 724.93 & 0.35 & 27.853 & 27.975 \\
\hline
\end{tabular}

bers and deformations. The oscillator constant was chosen as $\hbar \omega_{0}=7.55 \mathrm{MeV}$ in all cases. The results are presented in the first four columns of Tables 1 and 2. In Table 1 we show the case of $N=112$ particles for frequency ratios ranging from $q=0.4$ to $q=3.1$. In Table 2 the particle number is varied from $N=4$ to $N$ $=160$, this time at spherical shape $(q=1.0)$. In all cases we see that the energy $T[\tilde{\rho}]$ obtained through the functional $(4,5)$ agrees perfectly well with the Strutinsky averaged (or ETF model) quantity $\check{T}$ given by (15) and (9). The difference $\Delta T$ is in all cases well below $1 \mathrm{MeV}$, corresponding to an accuracy of about $10^{-4}$ for the heavier cases.

Concerning the numerical accuracy of these results, we have to remark that the plateau condition (14) does not hold as well for the energy $T[\tilde{\rho}]$ as for the directly calculated encrgy $\tilde{T}(15)$. Usually, both quantities have a stationary point for the same averaging width $\gamma_{0}$. Some minor differences were found, however, leading to an uncertainty in $T[\tilde{\rho}]$ of less than $\sim 0.5 \mathrm{MeV}$ in magnitude for all cases considered. Since the differences $\Delta T$ obtained lie within this limit, but otherwise seem rather random and without any systematic trend as function of $N$ and $q$, we can thus entirely attribute them to these plateau uncertainties.

Consequently we may conclude that, as far as the integrated average kinetic energies $\tilde{T}$ are concerned, the semiclassical functional $\tau[\rho](4,5)$ is practically exact, even for extremely large deformations or very small nucleon numbers. This confirms the results obtained earlier for a deformed Woods-Saxon potential and shown in Fig. 1 above. The remaining difference $\Delta T$ found there may also be attributed (at least partially) to plateau uncertainties in the Strutinsky averaging, known to be of the order of $\sim 1-2 \mathrm{MeV}$ in deformed Woods-Saxon potentials [18].

It might be interesting to see different contributions to $T[\tilde{\rho}]$ according to (3), obtained for the deformed harmonic oscillator. This is shown in Fig. 2 for $N$ $=112$ nucleons as a function of the deformation parameter $q$. The three solid curves present the energies obtained with the functional $\tau[\rho]$ including the terms up to the order indicated by the suffix. We see that the contribution from $\tau_{4}[\tilde{\rho}]$ is still of the order of $\sim 10$ to $15 \mathrm{MeV}$ here. It is the more surprising that 


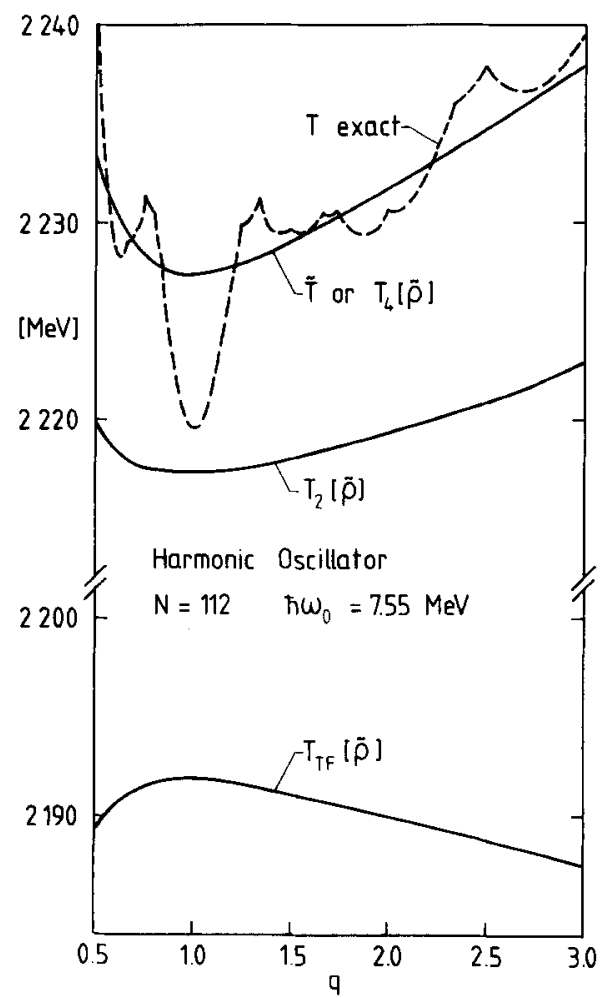

Fig. 2. The same as in Fig. 1, for 112 particles in an axially deformed harmonic oscillator potential with frequency ratio $q$ $=\omega_{1} / \omega_{z}$ (see text). The dashed curve shows the exact kinetic energy $T$

the sum of all terms reproduces the average kinetic energy $\tilde{T}$ so well. (Note that, as mentioned above, no further terms beyond $\tau_{4}[\rho]$ exist in the asymptotic expansion (3) if $\rho$ decays exponentially!)

In Fig. 2 we have also shown the exact kinetic energy $T$. The cusps occur wherever two levels cross at the Fermi energy. This curve demonstrates clearly our point made above that the local deformation behaviour of the exact energy $T(q)$ is completely different from that of the average energy $\tilde{T}$. In fact, since $N=112$ is a spherical magic number for the harmonic oscillator potential, the "branch" of the curve $T(q)$ in the range $0.833 \leqq q \leqq 1.2$ is given exactly by (7) with $\lambda \equiv q^{1 / 6}$. This leads, however, to a much larger curvature around the ground state $q=1.0$ than for the average energy $\tilde{T}(q)$. For the latter, it is also worth noting that the contribution from $\tau_{4}[\tilde{\rho}]$ is necessary not only to get the absolute average energy $\tilde{T}$, but also for obtaining its correct deformation dependence. Indeed, at e.g., $q=3$ about one half of the deformation energy, $\tilde{T}(q=3)-\tilde{T}(q=1) \simeq 10 \mathrm{MeV}$, comes from the fourth order term $\tau_{4}[\tilde{\rho}]$. The TF term alone would even lead to a negative kinetic deformation energy.

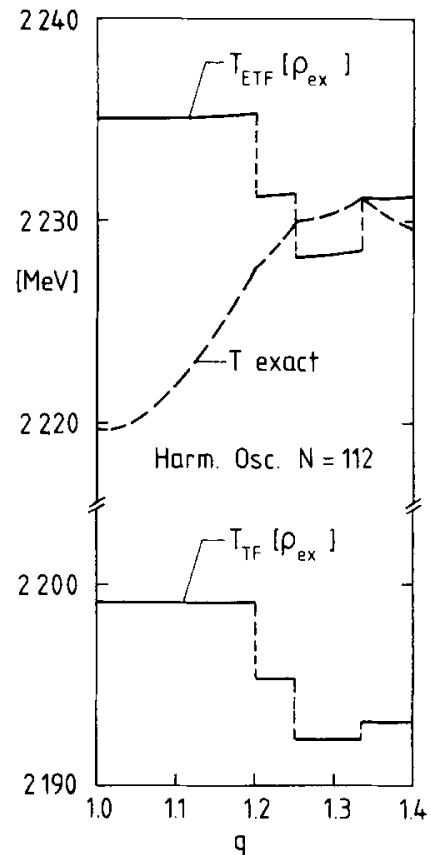

Fig. 3. Comparison of the Thomas-Fermi (TF) and extended Thomas-Fermi (ETF) kinetic energies $T[\rho]$ to the exact one $(T)$ when using the exact quantum-mechanical densities. Same case as in Fig. 2

That the exact kinetic energy $T(q)$ cannot be reproduced by the functional $\tau[\rho](4,5)$ using the exact density $\rho(r)$, is demonstrated in Fig. 3 for the same case. The complete functional is seen to overshoot the exact energy at $q=1.0$ by $\sim 16 \mathrm{MeV}$. The discontinuities in $T[q]$, at $q=1.2,1.25$ and 1.333 , stemming from the level crossings at the Fermi energy, are of course unrealistic. They would be smoothed out if some residual interactions, e.g., of BCS-pairing type, were included. Even then, however, the shell effects produced through the functional $\tau[\rho]$ have the wrong sign in this example.

This illustrates our statement 1) made above in Sect. II. It also puts some doubts on the way of readjusting the parameters of the truncated semiclassical functional $\tau_{\mathrm{TF}}[\rho]+\tau_{2}[\rho]$ in order to fit exact quantum-mechanical (Hartree-Fock) kinetic energies, as it was done in Refs. 10, 16. In particular, the requirement that their functional reproduce the exact kinetic energy for two nucleons in the $1 s_{1 / 2}$ state $\left(\mathrm{He}^{4}\right)$ leads their leading term to be zero for $N=2$ and smaller than the original TF term $\left(\tau_{\mathrm{TF}}\right)$ for all finite nuclei. As we clearly have shown in Table 2, there is absolutely no need for that. One consequence 


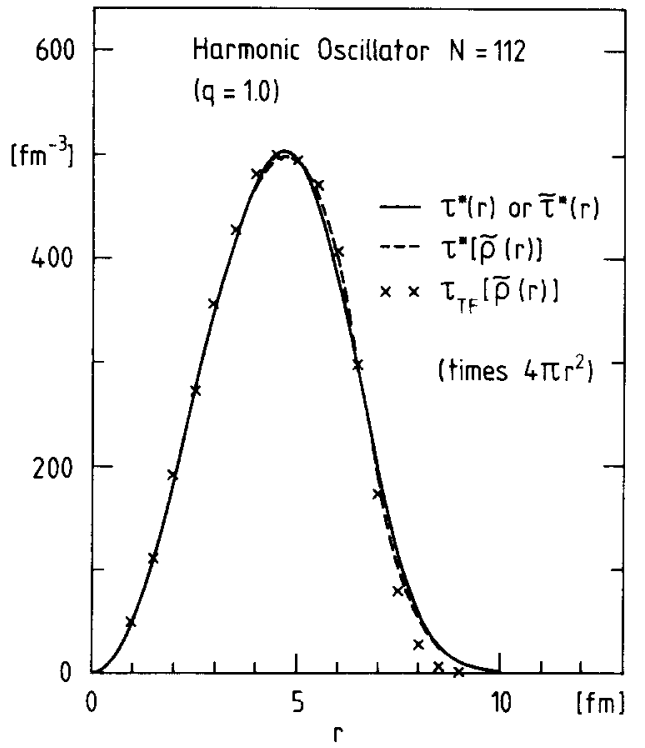

Fig. 4. Kinetic energy density distribution $\tau^{*}(r)(16)$ (with or without Strutinsky-averaging) compared to the result obtained through the TF or the full semiclassical ETF functional. All densities are multiplied by the volume element $4 \pi r^{2}$

of this procedure is that the functionals determined in $[10,16]$ cannot be used for deformed nuclei, neither in a quantum-mechanical nor a semiclassical description.

So far, we have only discussed the integrated kinetic energies. It might be interesting also to compare directly the distribution $\tilde{\tau}(r)$ to the one obtained with the functional (4). There is, however, one difficulty connected with such a comparison: There are oscillations present in the spatial distribution $\tilde{\tau}(r)$ which do not contribute to the kinetic energy after integration, and thus are quite spurious. A quantity that turns out to be completely smooth in $r$-space, even when calculated quantum-mechanically, is the following (cf. also [20]):

$\tau^{*}(r)=\tau(r)-\frac{1}{4} \Delta \rho(r)$.

In Fig. 4 we show the density distribution $\tau^{*}(r),(16)$, multiplied by the volume element $4 \pi r^{2}$, for the spherical case with $N=112$. It cannot be distinguished in this plot from the corresponding Strutinsky-averaged distribution $\tau^{*}(r)$. (I.e. the small differences leading to the shell correction $T-\tilde{T}=$ $-7.5 \mathrm{MeV}$ at $q=1.0$ after integration, lie within the thickness of the solid line.) The dashed curve in Fig. 4 shows the distribution $\tau^{*}[\tilde{\rho}(r)]$, obtained from (4) with the averaged densities $\tilde{\rho}(r)$ and subtracting $\frac{1}{4} A \tilde{\rho}(r)$ as in (16). We see that the two curves are very close over the whole region which contributes to the total integral. The crosses show the TF approxi- mation, $\tau_{\mathrm{TF}}[\tilde{\rho}]$, which is clearly too low in the tail region. The main difference between the latter and the full result $\tau^{*}[\tilde{\rho}]$ is brought about by the second terms, i.e. the Weizsäcker and the Laplacian corrections in $\tau_{2}[\tilde{\rho}]$. In fact, the contribution of $\tau_{4}[\tilde{\rho}]$ is less than $\sim 3 \%$ for $r \leqq 8 \mathrm{fm}$. Strictly speaking, we did not include the form of $\tau_{4}[\hat{\rho}]$ appearing in (4), but the integrand of the corresponding term in (5), which is easier to compute and gives identical contributions to the energy $T[\tilde{\rho}]$. This choice is, of course, somewhat arbitrary, but it seems justified to us by the smallness of that term.

In the far tail of the distribution $\tau[\tilde{\rho}]$, i.e., at $r \gtrsim 10 \mathrm{fm}$, the term $\tau_{4}[\tilde{\rho}]$ (or, respectively, the corresponding integrand of (5)) is the dominating one, due to its asymptotic form proportional to $\tilde{\rho}^{1 / 3}(r)$. This leads to an asymptotic fall-off which is too slow compared to that of $\tilde{\tau}(r)$. However, as we have seen in the above results (Tables 1, 2 and Fig. 2), this does not affect the integrated kinetic energies at all.

We have thus seen that the semiclassical functional $\tau[\tilde{\rho}]$ does not only lead to the exact average kinetic energies, but also to a very good local kinetic energy density distribution $\tilde{\tau}^{*}(r)$. To test other aspects of this same functional, we have also calculated the following moment of these distributions

$\left\langle r^{2}\right\rangle_{\bar{\tau}}=\frac{\int d^{3} r r^{2} \tilde{\tau}(r)}{\int d^{3} r \tilde{\tau}(r)}$

once using the Strutinsky averaged $\tilde{\tau}(r)$ and once using the functional $\tau[\tilde{\rho}]$ (4). The results are shown in the last two columns of Tables 1 and 2. The moment $\left\langle r^{2}\right\rangle_{i}$ tests the quality of the functional especially in the surface region. It might be expected that the too slow asymptotic fall-off of $\tau_{4}[\tilde{\rho}]$ leads to values that are too large. Still, the differences are seen to be less than $1 \%$ for $N \gtrsim 60$, which is rather satisfactory.

Another integral, which has more physical meaning, is the following one:

$\tilde{V}_{\tau}=C \int d^{3} r \tilde{\rho}(r) \tilde{\tau}(r)$.

It occurs as part of the potential energy in calculations with Skyrme interactions with an effective mass $m^{*}(r) \neq m_{0}$ [5]. A typical value for the coefficient $C$ in (18) for the interaction Skyrme III, e.g., is $C=\frac{1}{16}\left(3 t_{1}+5 t_{2}\right)=44.38 \mathrm{MeV} \mathrm{fm}^{5}$. We have computed this quantity, again using both $\tilde{\tau}(r)(12)$ and the functional $\tau[\tilde{\rho}]$ (4). The results $\tilde{V}_{\tau}$ and $V_{\tau}[\tilde{\rho}]$, respectively, and their differences $\Delta V_{\tau}$ are listed in Tables 1 and 2 . In all cases, the differences $\Delta V_{\mathrm{t}}$ are smaller than $\sim 0.6 \mathrm{MeV}$.

This test of the quantity $\tilde{V}_{\tau}(18)$ is not exactly consistent. As already stated in the beginning (point 7 in Sect. II), a Hamiltonian with variable effective mass 
$m^{*}(r)$ leads to further correction terms in the functional $\tau[\rho]$ depending explicitly on the gradients of $m^{*}(r)$. These corrections were derived up to second order in [8] and to 4th order in [13]. They would have to be included in a consistent test of a quantity like $\tilde{V}_{\tau}$ (18). But then, one also has to solve the Schrödinger equation with variable mass $m^{*}(r)$, which in 3 dimensions only can be done numerically. Such investigations will be the object of further studies. Irrespectively of that, the energy $\tilde{V}_{\tau}(18)$ as we have calculated it here represents a typical quantity which must be reproduced by the functional $\tau[\rho]$ along with the kinetic energy. It might be interesting to note that, in calculating $V_{\tau}[\tilde{\rho}]$, the term $\tau_{4}[\tilde{\rho}]$ contributed less than $0.2 \mathrm{MeV}$ in all cases considered. This may give hope that also in consistent calculations with effective masses $m^{*}(r) \neq$ const, the rather numerous terms in the complete 4 th order functional $\tau_{4}[\rho]$ given in [13] may be neglected in practical applications. The same conclusion has, in fact, been drawn by the authors of [13] from their calculations of surface energy coefficients using the nonlocal functional.

\section{Variational Euler-Lagrange Equation}

We now want to discuss briefly the contributions of the semiclassical functional $\tau[\rho]$ (4) to the variational equation obtained by minimizing the energy with respect to variations in $\rho(r)$. Although there exists no analogue to the Ritz principle for variations with respect to the density $\rho(r)$ in connection with approximate functionals $\tau[\rho]$, this method is quite familiar in nuclear physics since over forty years $[12,15,9]$. It was recently applied to Skyrme forces by the Orsay group (Bohigas et al. [1] and [3, 16]), but the term $\tau_{4}[\rho]$ was left out by these authors.

Let us assume for simplicity that the total nuclear potential energy is given as a functional of the density $\rho(r)$ only (as for a Skyrme force with constant nucleon mass), and treat one kind of nucleons only:

$E_{\mathrm{POT}}=\int d^{3} r \varepsilon_{\mathrm{POT}}[\rho(r)]$.

Including a Lagrange multiplier $\lambda$ for the particle number conservation, one minimizes then

$\frac{\delta}{\delta \rho} \int d^{3} r\left[\frac{\hbar^{2}}{2 m} \tau[\rho]+\varepsilon_{\mathrm{POT}}[\rho]-\lambda \rho\right]=0$.

The variation of the potential energy density gives, by definition, just the nuclear potential $V[\rho]$ (as a functional of $\rho$ again):

$\delta \varepsilon_{\mathrm{POT}}[\rho]=V[\rho] \delta \rho$.
Inserting the functional $\tau[\rho]$ (4) and performing the variation, we get after some partial integrations the following Euler-Lagrange equation:

$$
\begin{aligned}
& V[\rho]+\frac{\hbar^{2}}{2 m}\left\{\left(\frac{3 \pi^{2}}{2}\right)^{2 / 3} \rho^{2 / 3}+\frac{1}{36}\left[\left(\frac{V \rho}{\rho}\right)^{2}-2 \frac{\Delta \rho}{\rho}\right]\right. \\
& \left.+\left(\frac{3 \pi^{2}}{2}\right)^{-2 / 3} \rho^{-2 / 3} \frac{1}{6480} D^{4}[\rho]\right\}-\lambda=0,
\end{aligned}
$$

where

$$
\begin{aligned}
& D^{4}[\rho]=-176\left(\frac{\nabla \rho}{\rho}\right)^{4}+\frac{544}{3}\left(\frac{\Delta \rho}{\rho}\right)\left(\frac{\nabla \rho}{\rho}\right)^{2} \\
& +112 \frac{\nabla \rho \cdot \nabla(\nabla \rho)^{2}}{\rho^{3}}-66\left(\frac{\Delta \rho}{\rho}\right)^{2}-106 \frac{\nabla \rho \cdot \nabla(\Delta \rho)}{\rho^{2}} \\
& -27 \frac{\Delta(\nabla \rho)^{2}}{\rho^{2}}+48 \frac{\Delta^{2} \rho}{\rho} .
\end{aligned}
$$

(Here $\rho_{p}=\rho_{n}=\frac{1}{2} \rho$ !) A numerical solution of this fourth order, nonlinear equation is presently under investigation. It is easy to determine the asymptotic solution of (22) for large distances, where both $\rho(r)$ and $V(r)$ go to zero. The only way to make the fourth order contribution in (22) to be finite (and, in fact, asymptotically equal to the constant $\lambda$ ), is a solution for $\rho(r)$ which goes like

$\rho(r) \underset{r \rightarrow \infty}{\longrightarrow} \alpha r^{-6}$

if spherical symmetry is assumed. Putting in all the coefficients (and $\hbar^{2} / 2 m=41.46 \mathrm{MeV} \mathrm{fm}^{2}$ ), the constant $\alpha$ (in $\mathrm{fm}^{3}$ ) is given by

$\lambda=-\frac{\hbar^{2}}{2 m}\left(3 \pi^{2}\right)^{-2 / 3} \frac{13}{45} \alpha^{-2 / 3}=-0.6257 \alpha^{-2 / 3}[\mathrm{MeV}]$.

This result seems rather interesting to us. If the fourth order term in the functional, $\tau_{4}[\rho]$, is omitted (i.e., $D^{4}[\rho]$ is put equal to zero), then the asymptotic solution of (22) is governed by the term in square brackets in (22), coming from the Weizsäcker correction. The asymptotic fall-off of the density with spherical symmetry is then well-known $[15,9]$ to be exponential:

$\rho(r) \underset{r \rightarrow \infty}{\longrightarrow} \frac{1}{r^{2}} e^{-\kappa r}$

with

$\kappa^{2}=-\frac{2 m}{\hbar^{2}} 36 \lambda$.

(Remember that $\lambda$ is the Fermi energy of the average system and thus always negative.) Now, as discussed already by Lombard [9] and Bohigas et al. 
[1], the value (27) of $\kappa$ is unrealistically large. As a consequence, in variational equations based on the TF plus Weizsäcker approximation for the functional $\tau[\rho]$, the resulting densities $\rho(r)$ always fall off too quickly in the outer tail region and lead to an overestimation of the kinetic energy (which is partially compensated by an overestimation of the potential energy). This defect of such variational calculations is well-known $[1,9]$. It has sometimes, and recently again $[3,10,16]$, been overcome by increasing the coefficient $\frac{1}{36}$ of the Weizsäcker correction in $\tau_{2}[\rho]$ by a factor $\sim 4$ to 9 . (In fact, the old Weizsäcker coefficient $\frac{1}{4}$ [12] would lead to a realistic exponential fall-off, see also $[1,9,15]$.)

It is therefore interesting to see that the fourth order terms of the functional $\tau_{4}[\rho]$, which so far have not been taken into account in any variational calculation, lead to a much slower fall-off of the density $\rho(r)$. In fact, the $r^{-6}$ power may even be too slow for a realistic density, depending on how far out in the tail it will be assumed. But at least, the inclusion of the fourth order corrections seems to correct the behaviour of the variational density in the right direction.

These conclusions will not be changed in realistic situations $\left(\rho_{n} \neq \rho_{p}\right.$; effective mass, spin-orbit and Coulomb terms included).

\section{Summary and Conclusions}

We have shown with model calculations using deformed harmonic oscillator densities, that the semiclassical functional $\tau[\rho]$ as obtained from the ETFmodel is able to reproduce very accurately the average kinetic energy of $N$ nucleons, when averaged densities $\tilde{\rho}(r)$ are used. The spread of the semiclassical values around the exact ones is of the order of magnitude of the plateau uncertainties which are inherent to the Strutinsky smoothing; the absolute errors are less than $1 \mathrm{MeV}$. Furthermore this excellent agreement is independent of the number of nucleons and independent of the deformation of the nuclear shape. We hope that we have finally put an end to the controversy about the validity of the functional $\tau[\rho]$ for deformed shapes. This functional is entirely valid in so far as it is used correctly: The input density should be free of shell effects and the resulting energy is, a fortiori, free of quantal effects.

The functional $\tau[\rho]$ has been shown to be very useful for obtaining a reasonable spatial distribution of the kinetic energy density $\tau(r)$ as well as the second moment $\int r^{2} \tau(r) d^{3} r$ which emphasizes the tail be- haviour. One known major failure of the functional $\tau[\rho]$ when limited to the $\hbar^{2}$ term is that the nuclear density distribution minimizing the energy, thus solution of the variational Euler-Lagrange equation, has a too steep fall-off in the surface. We have shown that asymptotically, the inclusion of the fourth order term in the kinetic energy functional leads to a much slower fall-off of the density. It is worth noting that in the restricted variational calculations of [14], we have already observed this behaviour. There, the density distribution was fixed to be of the Fermi-type and its parameters were determined by minimizing the total energy. The diffuseness parameter was $0.41 \mathrm{fm}$ when the expansion was up to the $h^{2}$ term only and became $0.51 \mathrm{fm}$ (very close to experimental values) when the $\hbar^{4}$ terms were included. Similar results were also obtained for semi-infinite nuclear matter calculations by Grammaticos and Voros [13] using the functional $(3,4)$, restricting the shape of the density (i.e., the surface profile) to a Fermi function. These positive results for variations within a restricted space of densities will now have to be checked using the fully variational density which satisfies (22). Numerical calculations in this direction for the spherical case are presently in progress.

The case of velocity-dependent potentials has not been explicitly studied here. However, numerical calculations now under progress indicate that the inclusion of an effective mass and a spin-orbit potential does not make any problem.

In order to conclude, we would like to emphasize that the functional including the 4 th order terms is very well suited for calculating both smoothed total energies and spatial distributions of $\tau$. It is worth noting that the inclusion of the $\tau_{4}$ term does not bring any severe complication for the energy calculation; this just requires to know the quantities $\Delta \rho$ and $(V \rho)^{2}$. This functional has the main advantage of having no adjustable parameter and is valid for deformed shapes. There is thus no need for introducing phenomenological parameters $[10,16]$ which restrict the validity to spherical deformations.

The simplicity of the functional $\tau[\rho]$ makes it a powerful tool for calculating average deformation energies (as involved in fission) starting from effective forces of the Skyrme type [14]. Thus, it permits to study by numerically simple and fast methods the applicability of such forces to deformed nuclei, where constrained Hartree-Fock calculations are very time consuming.

We are grateful to Drs. Ph. Quentin and B.K. Jennings for useful comments. One of us (C.G.) likes to thank Prof. W. Weise for the warm hospitality extended to him at the Institute of Theoretical Physics in Regensburg. 


\section{References}

1. Bohigas, O., Campi, X., Krivine, H., Treiner, J.: Phys. Lett. B 64, 381 (1976)

Chu, Y.H., Jennings, B.K., Brack, M.: Phys. Lett. B 68, 407 (1977)

Chu, Y.H.: Ph.D. Thesis, S.U.N.Y. Stony Brook (1977)

Grammaticos, B.: Ann. Phys. (NY) 126, 450 (1980)

2. Holzwarth, G., Eckart, G.: Nucl. Phys. A 325, 1 (1979)

Eckart, G., Holzwarth, G.: preprint Siegen Si-79-8

3. Krivine, H., Treiner, J., Bohigas, O.: Nucl. Phys. A 336, 155 (1980)

4. Brack, M., Quentin, P.: Phys. Lett. B 56, 421 (1975)

5. Vautherin, D., Brink, D.: Phys. Rev. C 5, 626 (1972); see also Quentin, P., Flocard, H.: Ann. Rev. Nucl. Part. Sci. 28, $523(1978)$

6. Hohenberg, P., Kohn, W.: Phys. Rev. B 136, 864 (1964)

7. Kirzhnits, D.A.: Field Theoretical Methods in Many-Body Systems. Oxford: Pergamon Press 1967

Hodges, C.H.: Can. J. Phys. 51, 1428 (1973)

8. Jennings, B.K.: Ph.D. Thesis. McMaster (1976)

Brack, M., Jennings, B.K., Chu, Y.H.: Phys. Lett B 65, 1 (1976)

9. Lombard, R.: Ann. Phys. (N.Y.) 77, 380 (1973)

10. Campi, X., Stringari, S.: Nucl. Phys. A 337, 313 (1980)

11. Bhaduri, R.K., Ross, C.K.: Phys. Rev. Lett. 27, 606 (1971) Jennings, B.K., Bhaduri, R.K., Brack, M.: Nucl. Phys. A 253, 29 (1975)

12. v. Weizsäcker, C.F.: Z. Physik 96, 431 (1935)
13. Grammaticos, B., Voros, A.: Ann. Phys. (N.Y.) 123, 359 (1979) and preprint Saclay DPh-T/79/199 (1979)

14. Guet, C., Bengtsson, R., Brack, M.: Physics and Chemistry of Fission 1979. Proceedings of International Symposium at Jülich (I.A.E.A. Vienna, in print; paper I.A.E.A.-SM-241/H3) Guet, C., Håkansson, H.-B., Brack, M.: Preprint 1980. Phys. Lett. (submitted for publication)

15. Berg, R.A., Wilets, L.: Phys, Rev. 101, 201 (1956)

16. Krivine, H., Treiner, J.: Phys. Lett. B 88, 212 (1979)

17. Bohigas, O., Lane, A.M., Martorell, J.: Phys. Rep. 51, 267 (1979)

18. Brack, M., Pauli, H.C.: Nucl. Phys. A 207, 401 (1973)

19. Brack, M., Damgaard, J., Jensen, A.S., Pauli, H.C., Strutinsky, V.M., Wong, C.Y.: Rev. Mod. Phys, 44, 320 (1972)

20. Moszkowski, S.A.: Phys. Rev. C 2, 402 (1970)

21. Brack, M., Quentin, P.: Nucl. Phys. (submitted for publication)

22. Mermin, N.D.: Phys. Rev. A 137, 1441 (1965)

23. Jennings, B.K.: Preprint 1980. Phys. Lett. (submitted for publication)

C. Guet

M. Brack

Institut für Theoretische Physik

Universität Regensburg

Universitätsstraße 31

D-8400 Regensburg

Federal Republic of Germany

\section{Note Added in Proof}

The truncation of the functional $\tau[\rho]$ made in (3) after the term $\tau_{4}[\rho]$ - see the statement 3 ) in Sect. II. - is not necessary if the variational density $\rho(r)$ is used which satisfies the corresponding Euler-Lagrange equation. In fact, if terms up to order $\hbar^{2 n}$ are evaluated and $\tau_{\mathrm{ETF}}[\rho](3)$ is continued up to $\tau_{2 n}[\rho]$, the solution $\rho^{(n)}(r)$ of the variational equation will for $n \geqq 2$ go asymptotically like $\rho^{(n)}(r) \sim r^{-\alpha_{n}}$ with $\alpha_{n}=3 n /(n-1)$ for large $r$ (at spherical symmetry). In terms of this solution $\rho^{(n)}(r)$, the functional $\tau[\rho]$ does not diverge even for large $r$; the term $\tau_{2 n}\left[\rho^{(n)}\right]$ then goes in fact as $r^{-x_{n}}$, i.e., as $\rho^{(n)}(r)$ itself. (It remains to be checked numerically whether in this case the terms of order $n>2$ will be of any practical importance.) It is only with the use of model densities $\rho(r)$ with an exponential fall-off, that the terms beyond $t_{4}[\rho]$ diverge and must be left out. 